\title{
Belgeo
}

Revue belge de géographie

$4 \mid 2019$

Les politiques de mobilité urbaine en Europe après la crise : adaptations et innovations

\section{Financer un projet hors-norme : le nouveau réseau de métro du Grand Paris (2008-2018)}

\section{Alexandre Faure}

\section{(2) OpenEdition}

Journals

Édition électronique

URL : http://journals.openedition.org/belgeo/36271

DOI : $10.4000 /$ belgeo.36271

ISSN : 2294-9135

Éditeur :

National Committee of Geography of Belgium, Société Royale Belge de Géographie

Référence électronique

Alexandre Faure, «Financer un projet hors-norme : le nouveau réseau de métro du Grand Paris (2008-2018) », Belgeo [En ligne], 4 | 2019, mis en ligne le 04 février 2020, consulté le 20 juillet 2020. URL : http://journals.openedition.org/belgeo/36271; DOI : https://doi.org/10.4000/belgeo.36271

Ce document a été généré automatiquement le 20 juillet 2020

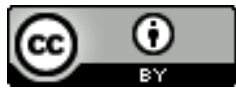

Belgeo est mis à disposition selon les termes de la licence Creative Commons Attribution 4.0 International. 


\title{
Financer un projet hors-norme : le nouveau réseau de métro du Grand Paris (2008-2018)
}

\author{
Alexandre Faure
}

\section{Introduction}

1 L'élection de Nicolas Sarkozy à la Présidence de la République française en 2007 marque un tournant pour la région parisienne. Le 17 septembre 2007, à la Cité de l'architecture et du patrimoine, il annonce le lancement du projet qu'il nomme Grand Paris. Celui-ci est composé de la création d'une série de clusters dont Paris-Saclay et la Défense sont les symboles, de la construction d'une grande infrastructure de transport en rocade (le Grand Paris Express) et de la réforme des institutions locales et départementales. La réalisation en est confiée à un secrétariat d'Etat spécifiquement créé pour l'occasion ayant pour objectif de trouver des solutions techniques (comment le projet sera matérialisé ?), politiques (quelles en seront les institutions porteuses ?) et budgétaires (comment financer en particulier le Grand Paris Express ?). Ainsi, le 18 mars 2008, Christian Blanc devient secrétaire d'Etat en charge du Développement de la région capitale. Ancien directeur d'Air France et de la RATP, son profil est sollicité par le Président de la République. Il avait notamment élaboré la dernière ligne de métro construite à Paris (la ligne 14, ouverte en 1998).

2 Un an après le discours de lancement du Grand Paris, le 15 septembre 2008, la banque Lehman Brothers se déclare en faillite. Une crise financière touche l'ensemble de l'économie mondiale. Cette situation de crise va servir à Nicolas Sarkozy pour appuyer son projet et notamment pour rendre légitime un investissement majeur dans l'infrastructure du Grand Paris Express (GPE). Le 29 avril 2009, le Président donne un nouveau discours à la Cité de l'architecture et du patrimoine au cours duquel il convoque à plusieurs reprises la crise et la lie à une opportunité de relancer la croissance française ${ }^{1}$. 
3 Pour aider Christian Blanc dans sa tâche, le député de la majorité présidentielle du Perreux-sur-Marne (banlieue est parisienne dans le département du Val-de-Marne) et spécialiste des finances, Gilles Carrez, reçoit la mission de rédiger un rapport visant à présenter des pistes de financement pour le GPE. Ce rapport remis au Premier ministre le 30 septembre $2009^{2}$ dessine la base du mode de financement de la nouvelle infrastructure qui sera adoptée dans la loi relative au Grand Paris.

4 Cette loi est présentée en 2009. L'argument de la relance économique autour de la centralité parisienne est utilisé par Christian Blanc lors de sa présentation au cours de la discussion générale le 6 avril 2010 au Sénat ${ }^{3}$. La crise financière est ici un argument pour promouvoir le projet du Grand Paris à la fois par le biais de son initiateur, Nicolas Sarkozy et par celui de son architecte, Christian Blanc.

« Il faut mettre le Grand Paris au cœur de notre stratégie de relance économique. Il nous faut soutenir l'activité en préparant l'avenir? Alors faisons-le avec le Grand Paris. La crise, c'est l'occasion ou jamais de faire en dix ans ce qu'autrement on ne pourrait pas faire en quarante ans » (Nicolas Sarkozy, 29 avril 2009, discours à la Cité de l'architecture et du patrimoine).

«Mesdames, messieurs les sénateurs, le monde dans lequel nous vivons change très vite. Ces transformations suscitent des inquiétudes chez nos concitoyens, qui en mesurent précisément les risques, sans en saisir les éventuelles promesses. Ils ont l'impression que la France n'a plus de prise sur les évolutions du monde. Comme si nous étions collectivement incapables de répondre aux grandes questions qui se posent à nous : comment être compétitifs sans abdiquer notre modèle social? Comment développer notre industrie sans sacrifier notre environnement? Comment placer la culture et la qualité de vie qui caractérisent l'identité française au cœur de notre société ? Avons-nous les potentiels et les modes d'organisation qui nous permettent, collectivement, d'entrer dans le nouveau monde du XXI ${ }^{\mathrm{e}}$ siècle ?» (Christian Blanc, 6 avril 2010 lors de la discussion générale de la loi relative au Grand Paris au Sénat).

5 La loi relative au Grand Paris ne reprend pas l'ensemble des annonces présidentielles. Les clusters ont été remplacés par des Contrats de Développement Territorial (CDT) dont les signatures entre acteurs locaux, régionaux et ministères se sont étalées de 2013 à 2016. La réforme des institutions politiques est abandonnée. La loi crée cependant la Société du Grand Paris (SGP) en charge de la construction du GPE. Le tracé de l'infrastructure et son financement ne sont alors pas définitifs. Ils seront modifiés successivement par deux accords Etat-Région en 2011 et 2013. Entre 2014 et 2015 est discutée la création de la Métropole du Grand Paris (inaugurée en 2016), formant le volet institutionnel. En 2017 un rapport critique de la Cour des Comptes sur la SGP ${ }^{4}$ rendait incertain la mise en chantier et la livraison de l'ensemble du projet d'ici 2035. Ce montant et le calendrier ont finalement été validés suite à un nouveau rapport de Gilles Carrez en 2018 et par une annonce d'Emmanuel Macron, Président de la République.

6 Ainsi, par un jeu de circonstances et de volontés politiques, la région parisienne connaît désormais un plan d'investissement colossal depuis la crise, plan estimé à 38,5 milliards d'euros ${ }^{5}$. L'étude des comptes de la SGP et de son activité montre une augmentation très rapide des dépenses depuis 2010, approchant en 2019 les $4 \mathrm{mds} €$. Cette somme apparaît bien éloignée de l'idée d'une raréfaction des ressources publiques induite par la crise financière. De plus, il est intéressant de voir dans le détail que ce n'est pas l'État à proprement parler qui s'endette pour construire le Grand Paris. En effet, l'ingénierie financière mise en place repose sur un système fiscal singulier, composé de taxes 
régionales devant permettre le remboursement d'emprunts souscrits par la SGP avec la garantie de l'État. Deux questions découlent de l'énoncé de ce contexte : comment un projet d'une telle ampleur a-t-il pu émerger au moment de la crise ? Et à quel stade de sa réalisation en est-on dix ans après?

7 Nous chercherons ainsi à expliquer le montage budgétaire et financier du GPE. En effet, le mode de financement du GPE est un objet d'étude utile à la compréhension de l'évolution du projet mais aussi à l'appréhension des enjeux politiques et urbanistiques de la région parisienne. Étudier le financement des infrastructures pour comprendre l'évolution des politiques publiques n'est pas une chose nouvelle.

Dans les études les plus spécialisées sur le financement des infrastructures de transport, il faut noter celles concernant les surcoûts liés aux grands projets d'infrastructure et notamment la modélisation de ces surcoûts qui ne nous concernera pas immédiatement (Flyvbjerg, 2007). Il y a aussi et surtout toute une littérature d'abord anglaise et américaine pour tenter d'expliquer le mode de financement des infrastructures de transports urbains à partir du contexte politique et social, local et national. Dans les années 1980, les changements politiques au Royaume-Uni entraînent une profonde transformation des financements publics. Pour trouver de l'argent, plusieurs pistes sont favorisées : la hausse des tarifs, la taxation de l'immobilier et du foncier à proximité des stations et enfin le recours à des investisseurs privés. Toutes ces solutions doivent permettre de pallier à la diminution des fonds publics (Kirwan, 1989 ; Bailey, 1991; Hart, 1993). Dans les années 2000, la financiarisation devient un enjeu central. Immobilier, foncier et infrastructures sont désormais des objets de recherche interconnectés (Leyshon et Thrift, 2007 ; Torrance, 2008; Aalbers, 2012 ; Guironnet et Halbert, 2014). Ce phénomène serait accentué dans les pays développés par l'action des gouvernements centraux (Gotham, 2006), mais aussi par les gouvernements locaux (Weber, 2010). Ce tournant de la financiarisation d'abord apparu au Royaume-Uni (Healey et al., 1992) s'est ensuite propagé aux Etats-Unis (Fainstein, 2001 ; Wolf-Powers, 2005). Il a pour conséquence de donner un rôle de plus en plus important au secteur privé dans le financement, la production et le fonctionnement des infrastructures de transports, particulièrement dans les villes globalisées (Fainstein, 2001 ; Pryke, 1994; Healey, 1994). Cette dynamique anglosaxonne est aussi visible dans le cas japonais (Aveline-Dubach, 2008 ; Sorensen et al., 2010).

9 Au-delà de ces approches, force est de constater qu'à Paris, l'avènement des acteurs privés dans la production des infrastructures du GPE n'a pas réellement été possible (exception faite de la ligne devant relier l'aéroport de Roissy à Paris mais dont la construction est encore incertaine). Si par le système de taxation, ce sont bien les entreprises qui vont finalement payer une grande partie de la nouvelle infrastructure, celle-ci sera entièrement publique. La crise financière de 2008 n'a pas changé fondamentalement le principe d'un financement public. Le ralentissement de l'économie perceptible dans les mois suivants a été utilisé comme un argument en faveur de ce projet et de son financement par les pouvoirs publics. Ce financement est le fruit d'une combinaison complexe croisant des contributions d'acteurs publics départementaux et régionaux, la création de nouvelles recettes régionales, la participation d'entreprises publiques de transport et la garantie de l'ensemble par le gouvernement central. C'est ici une particularité forte du Grand Paris Express. La diminution du rôle du gouvernement central s'est traduite par un soutien fort des collectivités territoriales et par une place accrue des parlementaires dans la définition 
du périmètre du projet et des outils de financement. Nous montrerons dans cet article que la multiplication des acteurs publics participant au projet à différentes échelles a permis de le consolider en se concentrant non pas seulement sur le coût final, mais sur la source de financement : un projet régional financé non par la nation entière mais par les acteurs économiques locaux.

\section{Résumé des dates importantes du projet d'infrastructure de transport du Grand Paris Express :}

$10-2007$ : Élection de Nicolas Sarkozy et discours de septembre sur les grandes lignes du Grand Paris

- 2008 (mars) : Nomination de Christian Blanc comme secrétaire d'État en charge du développement de la région capitale

- 2009 (avril) : Second discours de Nicolas Sarkozy sur le Grand Paris et lancement des discussions sur la loi relative au Grand Paris

- 2009 (septembre) : Remise du rapport sur le financement du Grand Paris par Gilles Carrez

- 2010 (juin): Vote de la loi relative au Grand Paris dont l'objet est seulement l'infrastructure de transport - Création de la Société du Grand Paris

- 2010 (juillet) : Démission de Christian Blanc et suppression du secrétariat d'État en charge du développement de la région capitale

- 2011 (janvier) : Accord État-Région sur le tracé du Grand Paris Express et son financement

- 2012 (décembre): Rapport de la mission sur le calendrier pluriannuel et de financement du projet du Grand Paris Express de Pascal Auzannet

- 2013 (juillet) : Accord État-Région dit du Nouveau Grand Paris révisant le tracé du Grand Paris Express, ajoutant de nouvelles infrastructures et confortant le financement

- 2013 (décembre): Approbation du Schéma Directeur de la Région Île-de-France comprenant le tracé du Nouveau Grand Paris

- 2016 (avril) : Démarrage des travaux du Nouveau Grand Paris

- 2017 (décembre) : Rapport critique de la Cour des Comptes sur le financement de la Société du Grand Paris

- 2018 (juillet) : Rapport sur les ressources de la Société du Grand Paris de Gilles Carrez

- 2018: confirmation par le Président de la République et le gouvernement de la construction de l'intégralité de l'infrastructure du Nouveau Grand Paris

11 Glossaire

CPER : Contrat de Plan Etat-Région

GPE : Grand Paris Express

NGP : Nouveau Grand Paris

RATP : Régie Autonome des Transports Parisiens

SDRIF : Schéma Directeur de la Région Ile-de-France

SGP : Société du Grand Paris

SNCF : Société Nationale des Chemins de Fer

STIF : Syndicat des Transports de la Région Île-de-France (aujourd'hui Île-de-France Mobilités) 
Figure 1. Carte des départements de la région Ile-de-France.

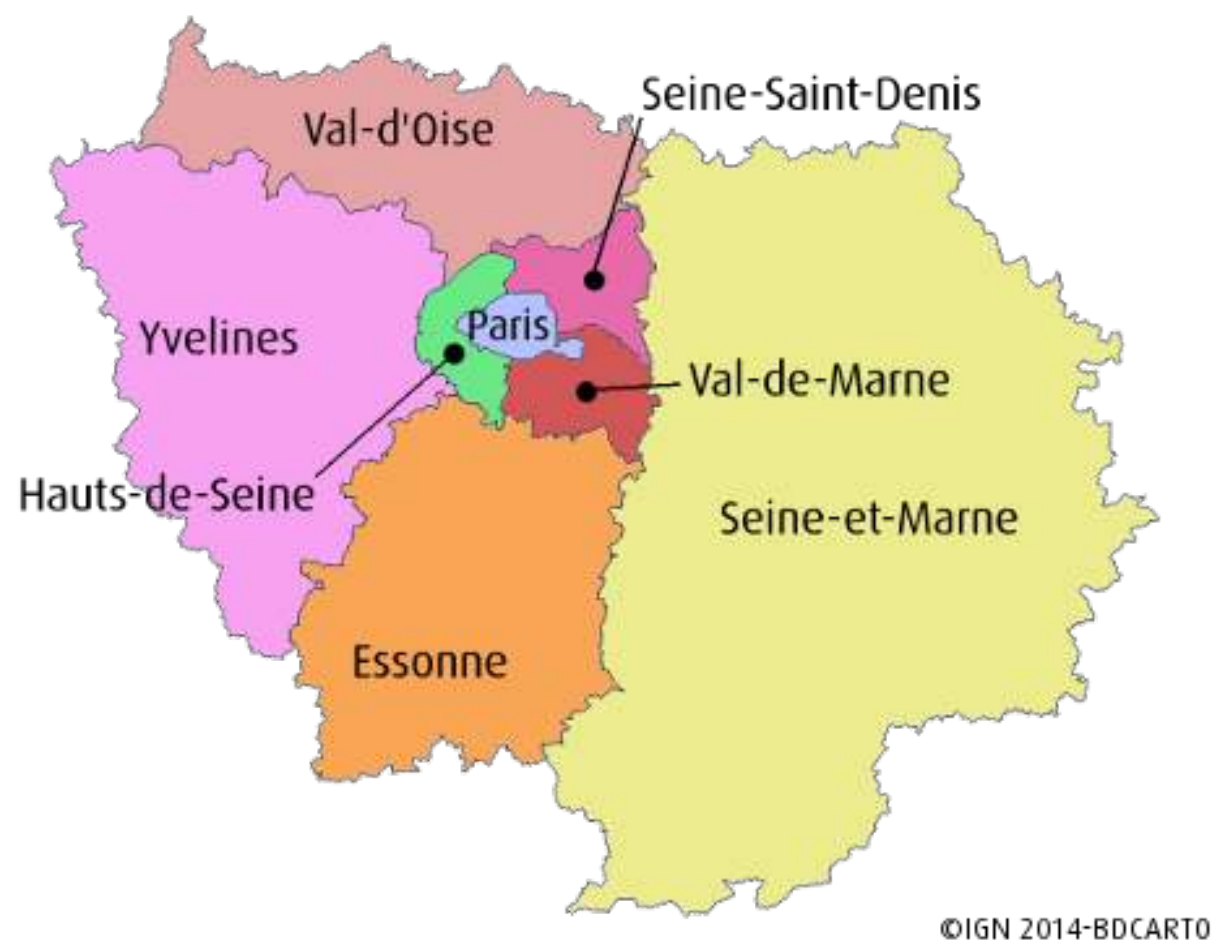

Figure 2. Carte de la Métropole du Grand Paris instaurée en 2016.

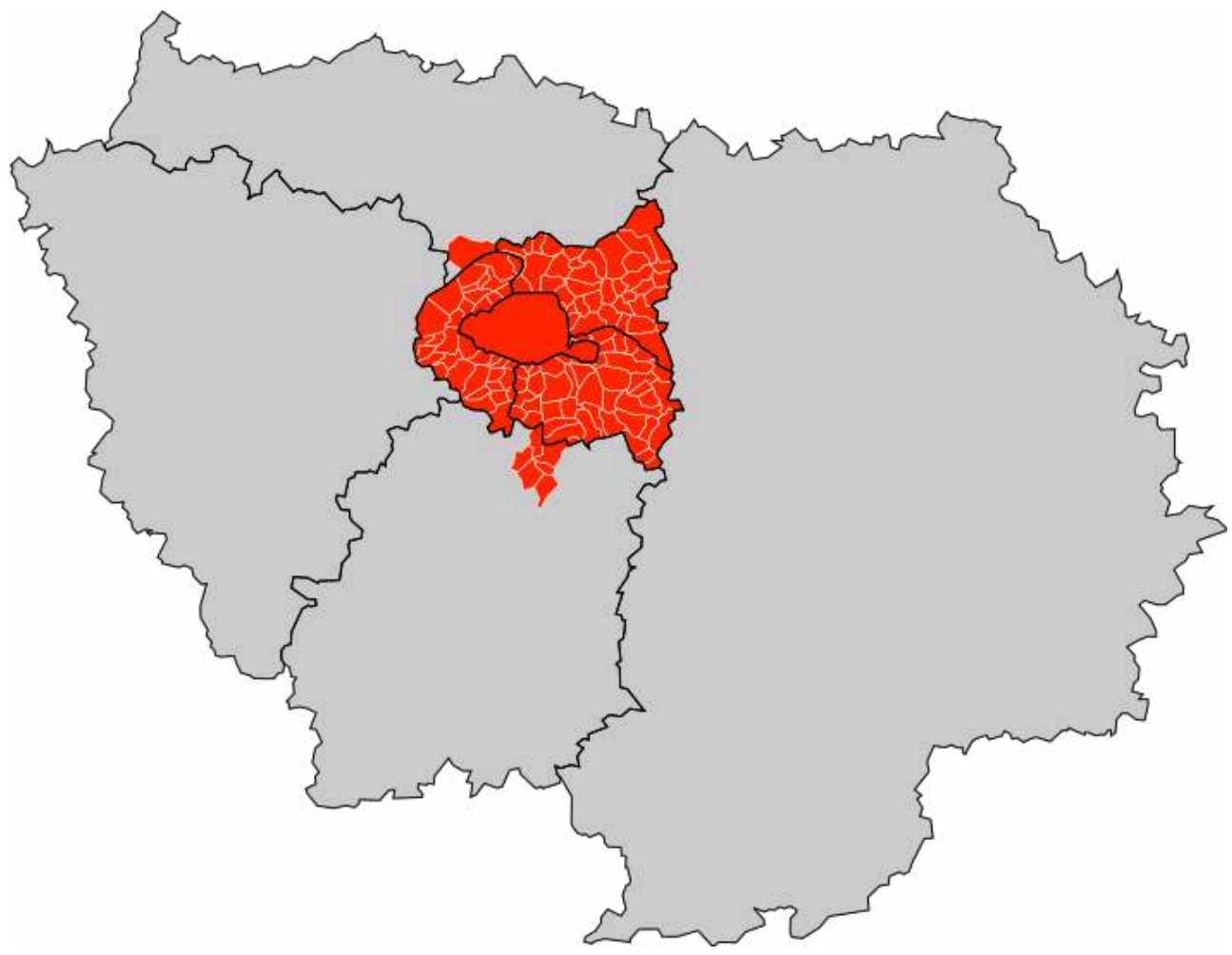

Wilkipedia Commons, 2016 


\section{4-2007 : multiplication des projets d'infrastructures de transport en Ile-de-France} simultanément: locale, régionale et nationale. Au niveau national, la loi d'Orientation des Transports Intérieurs de 1982, avait profondément modifié la gouvernance des transports publics urbains en décentralisant leur gestion au niveau des collectivités territoriales (LOTI : titre III, article 46, page 4010 du JO). Cependant, cette loi n'a pas concerné la région Île-de-France. C'est en 2000 que le Syndicat des Transports de la région Île-de-France (STIF aujourd'hui Île-de-France Mobilité - IDFM) est créé sous l'autorité du gouvernement central. En 2006, son contrôle est transféré au conseil régional dont les représentants obtiennent une majorité absolue de sièges au conseil d'administration.

13 À partir de 2004, plusieurs projets d'infrastructure de transport émergent. La Régie Autonome des Transports Parisiens (RATP - entreprise publique gestionnaire du réseau de métro, d'une partie des RER A et $\mathrm{B}$, des tramways et des bus en première couronne) présente en 2004 une réécriture d'un vieux projet nommé métropériphérique (Auzannet, 2018), préalablement connu sous le nom d'Orbital. Ce projet proposait la construction d'une rocade de métro (Zentelin, 2010), permettant d'améliorer les connexions entre banlieues en désengorgeant les interconnexions centrales (figure 3). Quelques mois plus tard, c'est au conseil général du Val-de-Marne de proposer un projet de ligne de métro traversant le département et nommé Orbival (figure 4). Enfin, le conseil régional propose de son côté Arc Express, deux grandes lignes au nord et au sud de Paris, en première couronne, devant relier l'est et l'ouest de l'agglomération. Ce dernier projet évoluera pour devenir une rocade en 2007 (figure 5).

Il faut ajouter deux autres points importants concernant les transports franciliens. Tout d'abord la signature du Contrat de Plan État Région (CPER) 2007-2013 qui intègre de nombreux financements pour développer des réseaux de transports dans l'ensemble de la région. Il affiche une volonté de continuer les investissements dans les lignes de tramways en banlieue, de prolonger certaines lignes de métro existantes ou d'aménager des lignes de bus en site propre. De fait, le CPER suit en partie les orientations prises lors de la révision du Schéma Directeur de la Région Île-de-France par le conseil régional entre 2004 et 2007. Pour autant, le SDRIF intègre dans ces orientations la construction d'Arc Express, ce que le CPER ne prévoit pas de financer, reléguant ainsi ce projet à une simple annonce sans moyens effectifs.

C'est donc dans ce contexte de concurrence entre institutions locales, régionales et nationales pour la définition d'un projet métropolitain d'infrastructure de transport que s'ouvre la séquence électorale de 2007 voyant Nicolas Sarkozy, Président du conseil général des Hauts-de-Seine, devenir Président de la République (Faure, 2018). 
Figure 3. Carte du Métropériphérique.

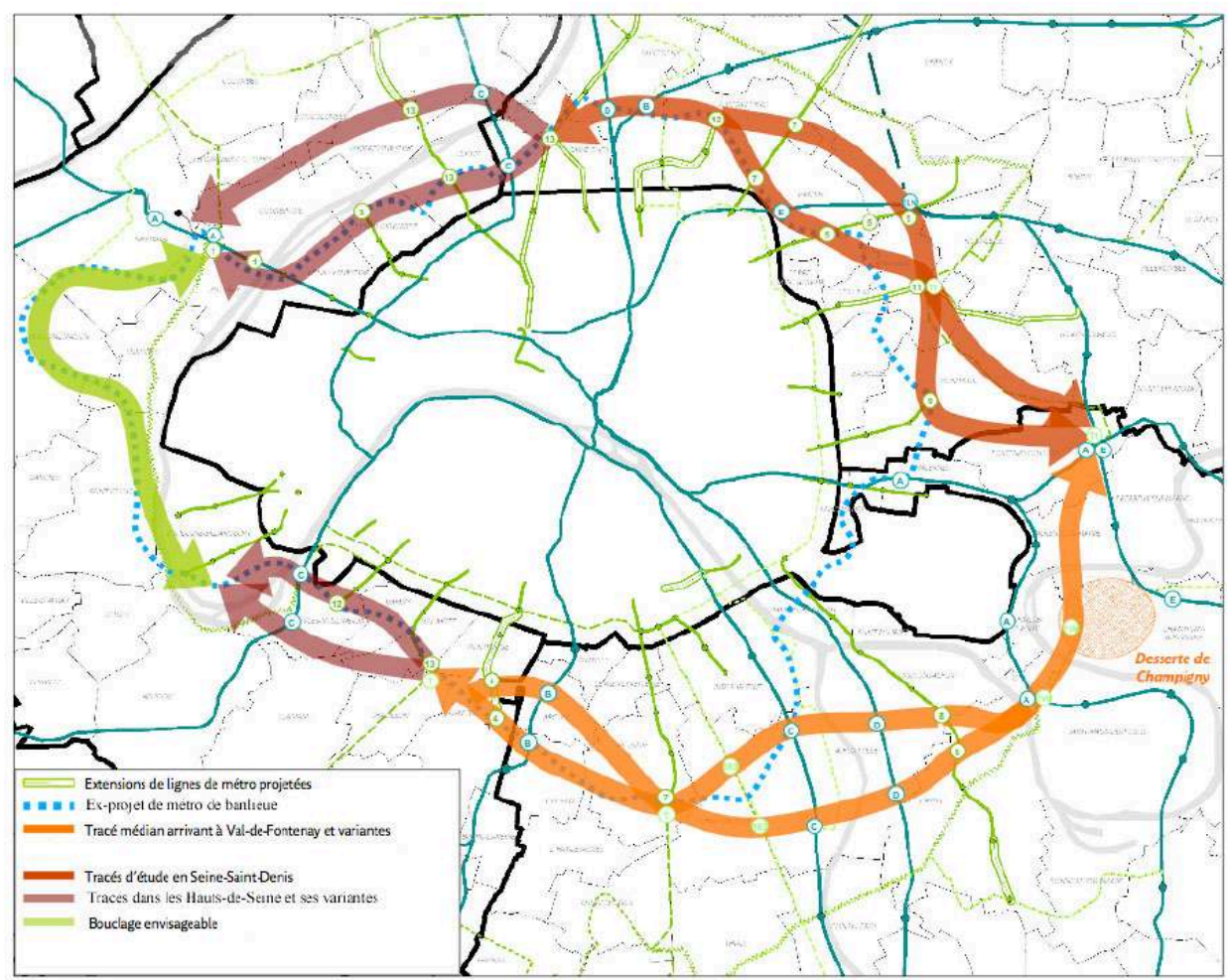

RATP, 2004

Figure 4. Carte d'Orbival.

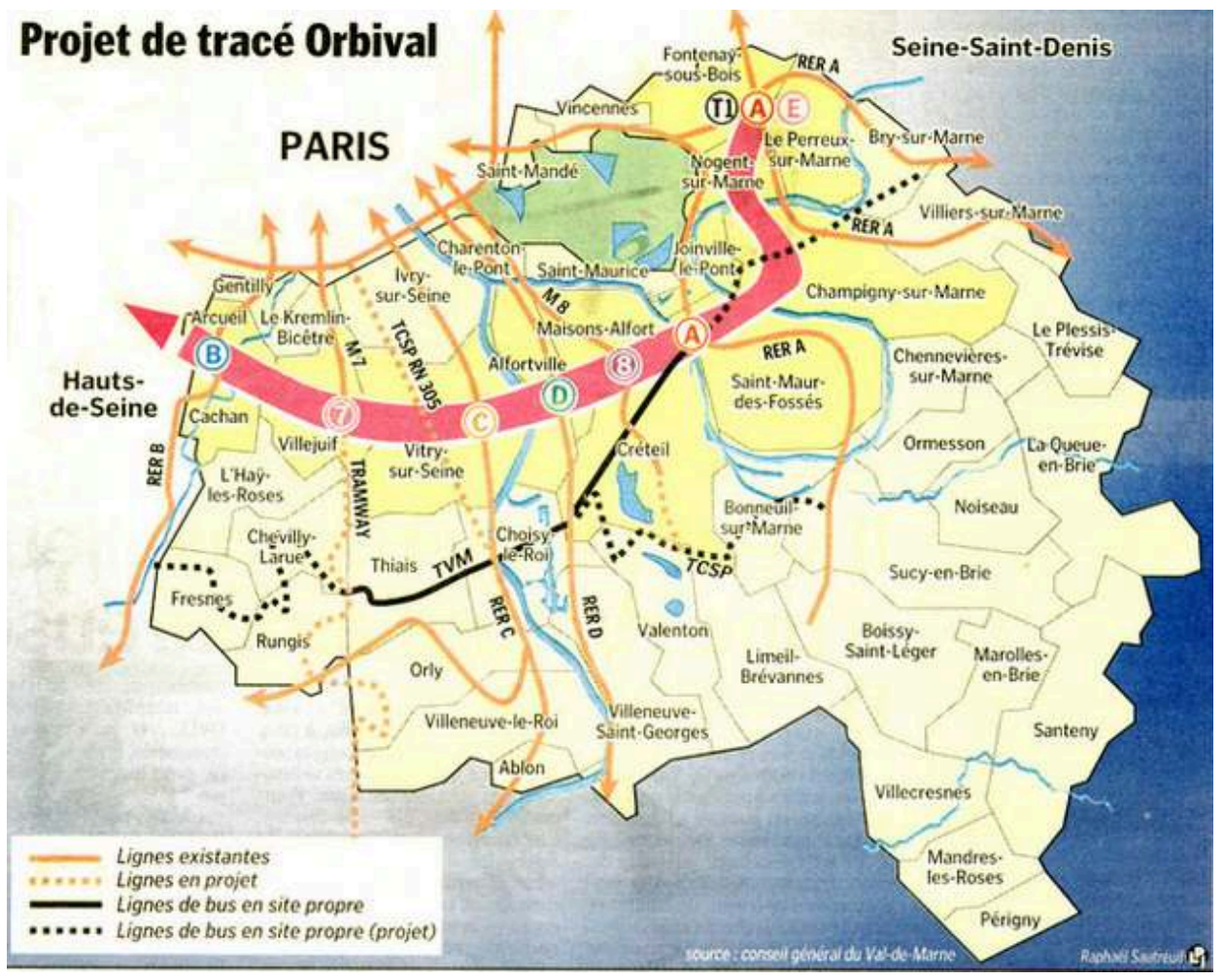

Conseil général du Val-de-Marne, 2006 
Figure 5. Carte d'Arc-Express.

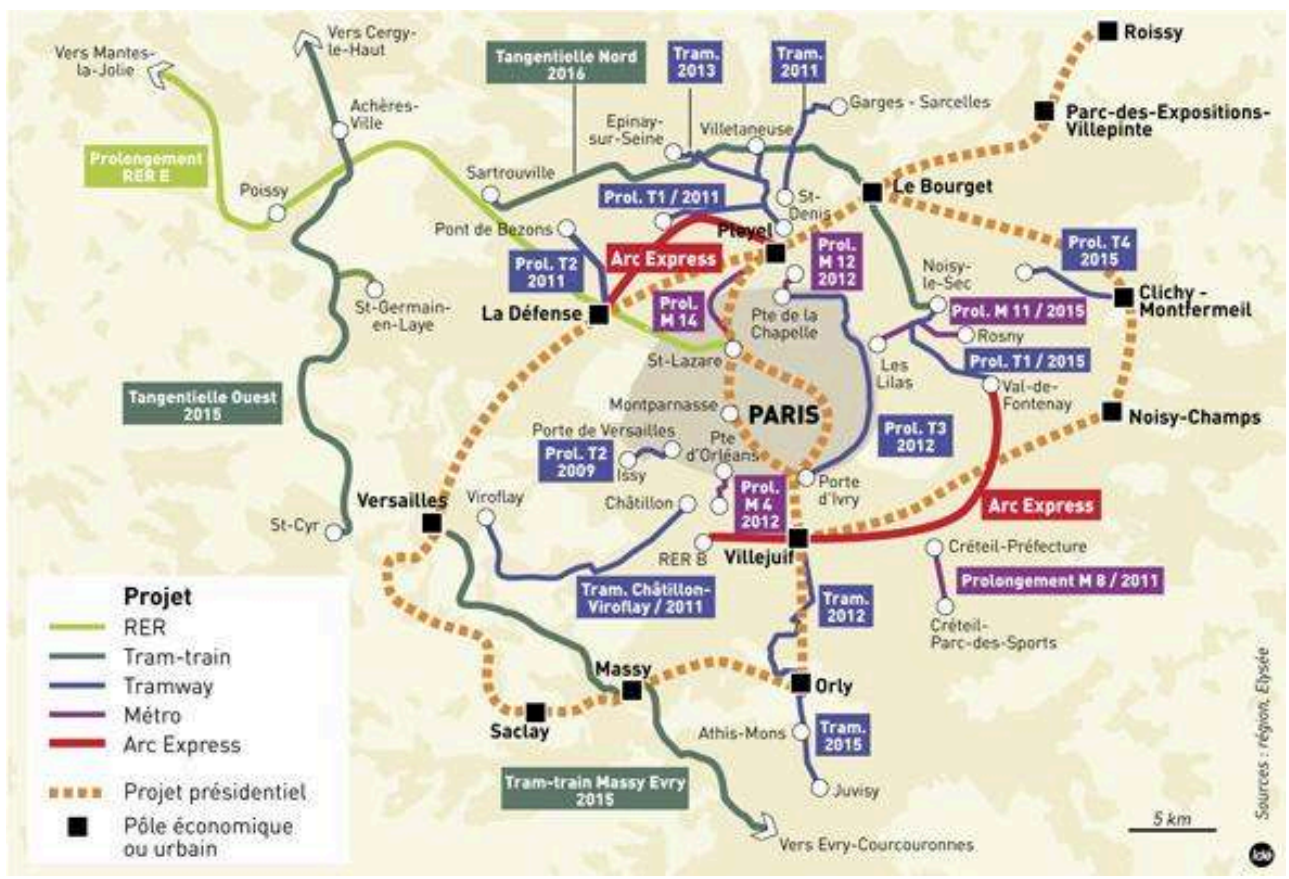

Urba-news.fr, 2007 : https://www.urbanews.fr/wp-content/uploads/2009/11/article_2904-PARprojetsGrand-Paris.jpg

\section{Du Grand Paris Express au Nouveau Grand Paris : création d'un consensus régional}

Le Grand Paris de Nicolas Sarkozy se définit par une grande rocade de métro en banlieue reliant des clusters avec le centre de Paris et les aéroports. «À ce stade du projet du Grand Paris, nous avons identifié neuf territoires de projet, qui seront les grands axes du développement de la région-capitale ${ }^{6}$. Ces clusters concernent principalement la recherche (Saclay, Vallée de la Bièvre, Condorcet) et la finance (La Défense). Ils doivent permettre de relancer la croissance économique de Paris et de la France. In fine, comme l'indiquent plusieurs auteurs, ce projet a pour conséquence de concentrer plus encore la finance et la recherche en région parisienne (Gilli, 2014 ; Orfeuil et Wiel, 2012), dynamique jugée nécessaire par les décideurs nationaux participants à ce projet pour améliorer l'attractivité et la compétitivité de la métropole parisienne dans le cadre de la compétition internationale des villes (Bouba-Olga et Grossetti, 2008). 
Figure 6. Carte du Grand Paris Express.

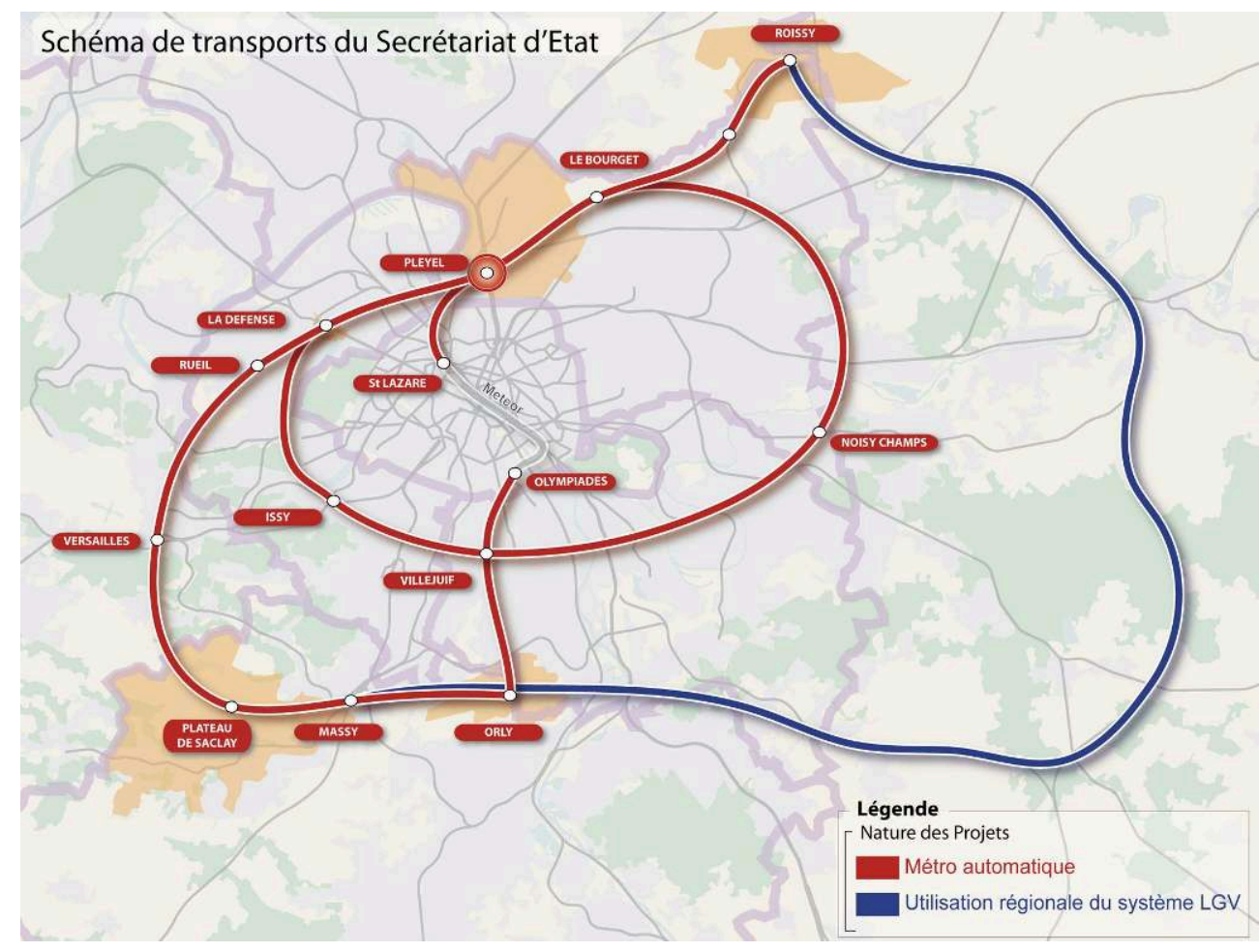

Rapport Carrez, 2009

La loi relative au Grand Paris donne à la Société du Grand Paris, qu'elle crée, la mission de réaliser l'infrastructure. Cependant, suite au départ de Christian Blanc en 2010, de nouvelles négociations avec le conseil régional ont lieu aboutissant à un accord EtatRégion en janvier 2011 dont le résultat est une augmentation du nombre de stations prévues. Peu après cet accord, la droite perd aux sénatoriales (2011), à la présidentielle et aux législatives (2012). Le personnel politique change au niveau national et plusieurs élus franciliens intègrent des ministères (Manuel Valls - Parti Socialiste, Cécile Duflot Europe-Ecologie Les Verts, et François Lamy - PS). Ces élus locaux aux positions nationales vont négocier avec Jean-Paul Huchon, Président PS du conseil régional, un nouvel accord, nommé Nouveau Grand Paris (NGP). Cet accord signé en 2013 prévoit à nouveau une augmentation du nombre de stations, une confirmation des financements inscrits dans la loi de 2010, la participation de la SGP au plan de mobilisation pour les transports franciliens (le plan de mobilisation vise à accélérer la modernisation du matériel roulant et des stations de transports) et l'accélération du calendrier en prévision de la candidature de la France et de Paris à l'obtention des Jeux Olympiques de 2024 et de l'Exposition Universelle de 2025. Finalement, cet accord confirme que la SGP construira le nouveau réseau, le financera avec un système d'emprunts et de réemprunts garantis par l'État, et donnera sa gestion au STIF et à la RATP une fois l'opération finalisée. L'ensemble des acteurs locaux, départementaux et régionaux soutiennent le programme d'investissement bien que des critiques et des conflits épars persistent. La confirmation du projet après l'alternance au niveau national entre 2011 et 2013 consolide l'ensemble alors même que le périmètre de financement du NGP s'avère beaucoup plus important que celui du GPE. 
Figure 7. Carte du Nouveau Grand Paris.

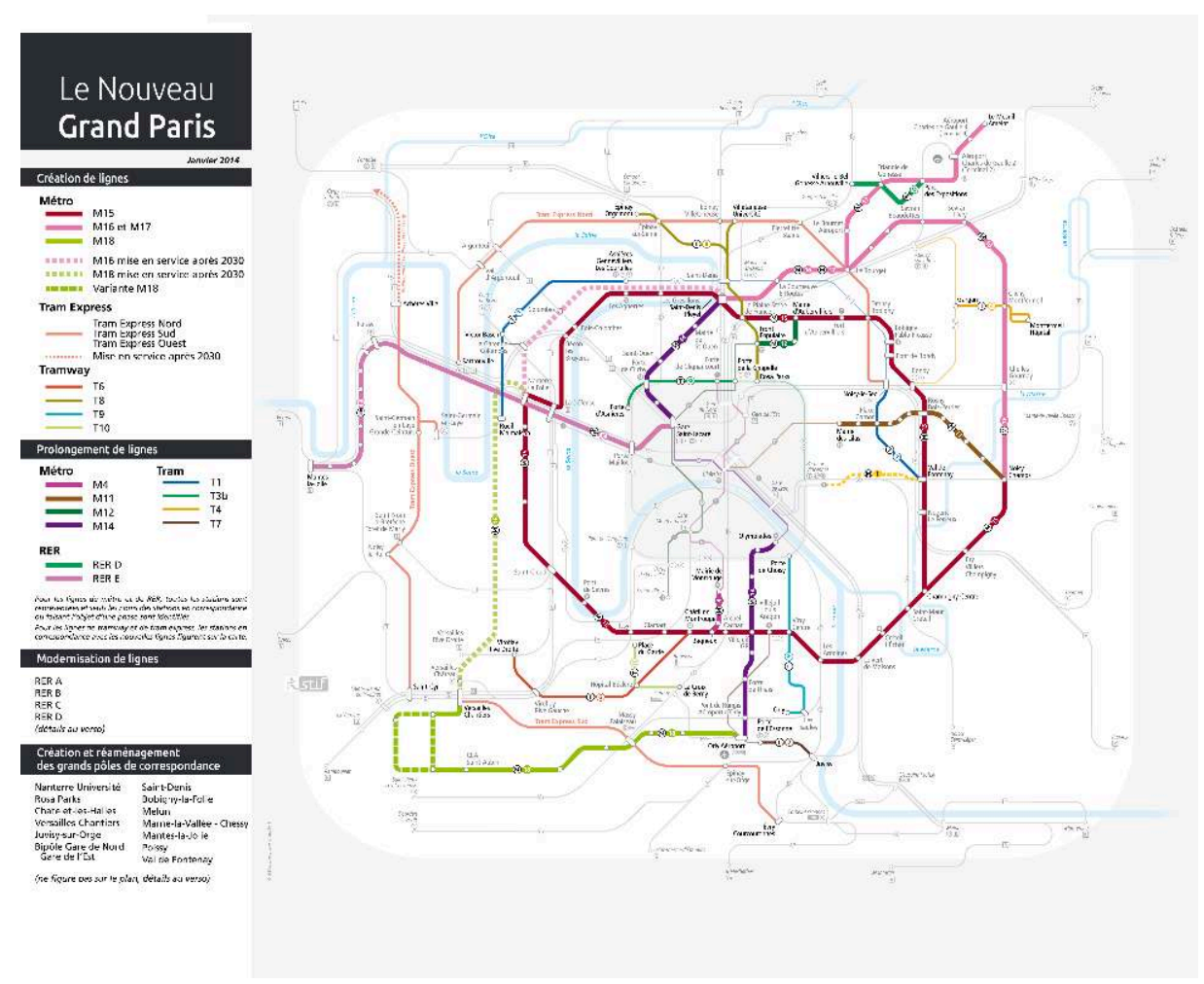

Société du Grand Paris, 2014

\section{Quelles dépenses prendre en compte?}

Pour prendre conscience de la place singulière que prend le Grand Paris Express dans ce mouvement de prolifération des projets d'infrastructures de transport en région francilienne, il est nécessaire de comparer les budgets de ces différents projets. Ainsi, le métropériphérique proposé par la RATP devait coûter selon les estimations entre 4 et 5 milliards d'euros, Orbival un peu moins de 2 mds $€$ et Arc Express entre 5 et 6 mds $€$ en 2007. De son côté, le Grand Paris de 2009 est estimé à $28,9 \mathrm{mds} €$. Les coûts du métropériphérique et d'Arc Express apparaissent proches, à l'image de leurs tracés. Orbival ne faisant pas une boucle et ne concernant qu'un des trois départements de première couronne coûterait environ un tiers des deux autres projets. Le coût du GPE apparaît donc en rupture avec les autres projets.

Tableau 1. Synthèse des coûts des différents projets.

\begin{tabular}{c|c|c|c|c|c|c|c}
\hline Date & $\mathbf{2 0 0 5}$ & $\mathbf{2 0 0 6}$ & $\mathbf{2 0 0 7}$ & $\mathbf{2 0 0 9}$ & $\mathbf{2 0 1 1}$ & $\mathbf{2 0 1 3}$ & $\mathbf{2 0 1 8}$ \\
\hline Nom & Métropériphérique & Orbival & Arc-Express & $\begin{array}{c}\text { Grand Paris } \\
\text { Express }\end{array}$ & Accord état Région & $\begin{array}{c}\text { Nouveau Grand } \\
\text { Paris }\end{array}$ & Grand Paris \\
\hline Porteur & RATP-SNCF & $\begin{array}{c}\text { Conseil Général du } \\
\text { Val-de-Mame }\end{array}$ & $\begin{array}{c}\text { Conseil Régional } \\
\text { d'lle-de-France }\end{array}$ & Gouvemement & SGP & SGP & SGP \\
\hline Coût en milliards d'euros & $\mathbf{5}$ & $\mathbf{1 , 8}$ & $\mathbf{6}$ & $\mathbf{2 8 , 9}$ & $\mathbf{3 2 , 4}$ & $\mathbf{2 6 , 6}$ & $\mathbf{3 8 , 5}$ \\
\hline Sources & RATP & $\begin{array}{c}20 \text { inites du } \\
\text { Christianterview Favier }\end{array}$ & $\begin{array}{c}20 \text { minutes } \\
\text { Ch/01/08 STIF et } \\
\text { RATP }\end{array}$ & $\begin{array}{c}\text { Rapport Carrez I } \\
\text { Raccord 2011 }\end{array}$ & Accord 2013 & Rapport Carrez II
\end{tabular}

Alexandre Faure, 2018 
Figure 8. Evolution des coûts estimés pour les infrastructures de transport dont la rocade autour de Paris.

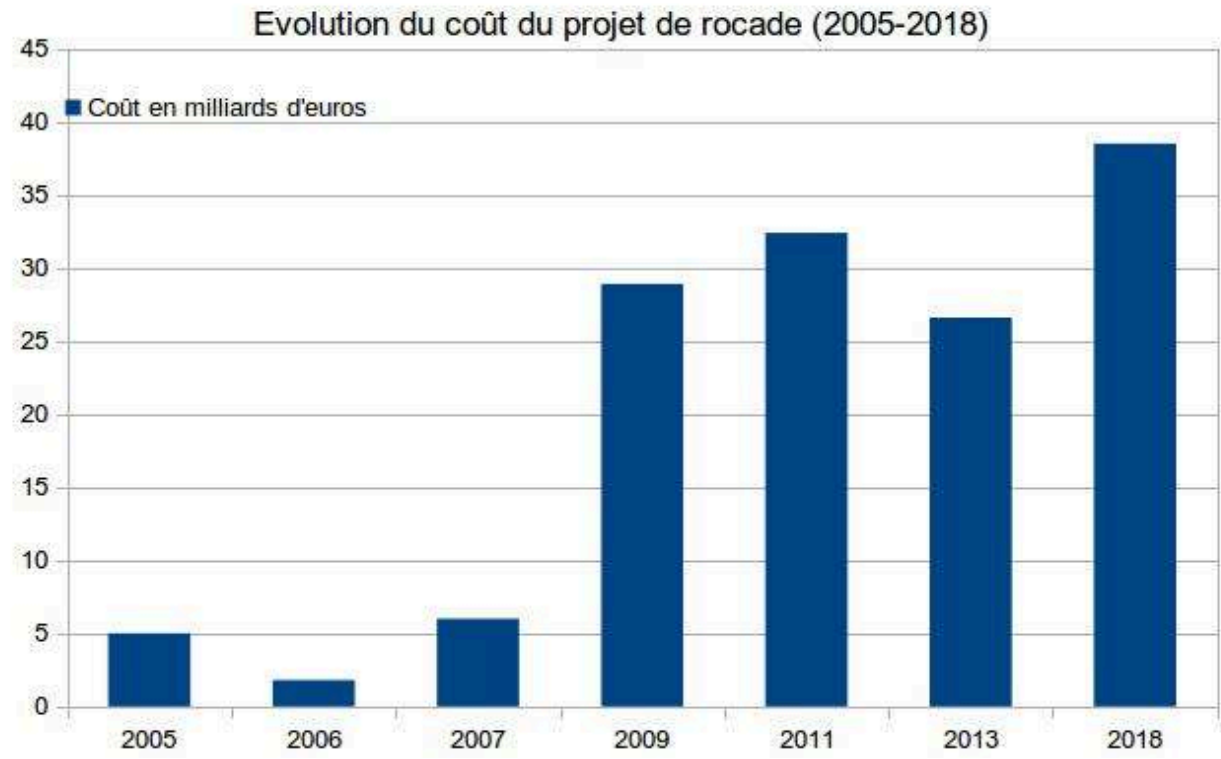

Alexandre Faure, 2018

La première raison de cette différence réside dans le périmètre du projet faisant l'objet de l'estimation. En effet, le rapport Carrez 2009 fait une estimation du Grand Paris en comprenant l'investissement dans l'infrastructure, les interconnexions, l'accessibilité du réseau pour les personnes à mobilité réduite, le matériel roulant, les charges d'exploitation supplémentaires pour le réseau actuel et le nouveau réseau ainsi que les coûts de l'ingénierie financière. Le rapport fait état d'un besoin d'investissements de 28,9 mds $€$ pour la seule construction de l'infrastructure qui est l'élément comparable avec ceux inclus dans les estimations pour les projets précédents. Afin de rendre compte de l'ensemble du périmètre pris en compte dans le rapport Carrez, le tableau cidessous montre les dépenses en investissement et en fonctionnement. La somme totale est de $72 \mathrm{mds} €$ en comprenant les $24,1 \mathrm{mds} €$ imputés à la dérive du socle de fonctionnement du réseau existant (augmentation des frais de fonctionnement du réseau qui n'est pas encore financée au moment de l'écriture du rapport). Le financement du matériel roulant $(9,2 \mathrm{mds} €$ ) et de l'accessibilité ( $1,8 \mathrm{mds} €)$ s'inscrivent simultanément dans la finalisation du CPER 2007-2013, dans le futur CPER (2015-2020), dans le plan de mobilisation pour les transports franciliens et dans le rapport Carrez sans qu'il soit précisé dans quelle mesure il revient à la SGP de prendre en charge ces éléments concernant le réseau existant. De fait, le rapport fait le choix d'élargir le financement du GPE à des domaines qui ne lui sont pas initialement dévolus.

Tableau 2. Dépenses prises en compte dans le rapport Carrez I de 2009 (en millions d'euros).

\begin{tabular}{|l|l|}
\hline Investissements dans les infrastructures - Phase I (2010-2025) & 24400 \\
\hline Investissements dans les infrastructures - Phase II (Au-delà de 2025) & 4500 \\
\hline Dérive du socle de fonctionnement du réseau existant (2010-2025) & 24100 \\
\hline
\end{tabular}




\begin{tabular}{|l|l|}
\hline Charges d'exploitation supplémentaires sur le nouveau réseau (2010-2025) & 8100 \\
\hline Rénovation et achat du matériel roulant (2010-2025) & 9200 \\
\hline Accessibilité des stations existantes (2010-2025) & 1800 \\
\hline Sous-total des investissements & 28900 \\
\hline Sous-total des besoins complémentaires & 19100 \\
\hline Total & 72100 \\
\hline
\end{tabular}

Comme nous le voyons, le rapport montre un faisceau de dépenses très large. Dans les propositions qu'il émet, il distingue ce qui relève des investissements purs (infrastructure, matériel roulant, interconnexion) et ceux qu'il considère comme complémentaires (fonctionnement du réseau, rénovation, accessibilité...). Dans ce cadre, la dérive du socle est une dépense complexe à prendre en compte. Le socle représente le fonctionnement du réseau existant. La dérive représente le taux d'augmentation de ces coûts qui sont supérieurs à l'inflation et supérieur aux recettes existantes et à leur évolution probable. Par ce biais, le rapport inscrit dans le financement du GPE l'augmentation du coût de fonctionnement du réseau existant. L'élargissement des dépenses prises en compte au-delà de la nouvelle infrastructure peut s'expliquer de plusieurs manières. Cela peut être dû à une mécompréhension de la lettre de mission, ou cela peut être une liberté prise par le rapporteur pour avancer certaines idées : le rapport propose, comme nous allons le voir à présent, des pistes de recettes nouvelles potentiellement difficiles à faire accepter. Par exemple, il propose de créer un péage urbain, une taxe sur les poids lourds ou encore de transférer une partie de la taxe sur les produits pétroliers.

\section{Quelles recettes envisageables?}

21 Au moment de l'écriture du rapport, le Grand Paris Express est encore au stade d'esquisse. On ne sait pas qui sera en charge de sa construction, avec quel argent et sur quel calendrier. L'objectif de ce rapport est donc d'explorer les différents scenarii possibles et d'en fixer les bases.

Deux éléments centraux ressortent: les recettes visées par le rapport sont très majoritairement franciliennes (Gilles Carrez considère que le projet n'est viable que s'il repose sur un financement local), les recettes supplémentaires doivent servir à rééquilibrer le développement régional en favorisant l'est de la première couronne (la plus pauvre) par rapport au centre et à l'ouest beaucoup plus riches. Il est nécessaire ici de rappeler que le projet du GPE a été lancé par Nicolas Sarkozy, ancien Président du conseil général des Hauts-de-Seine (département de l'ouest parisien le plus riche de la région) et que Gilles Carrez est député du Val-de-Marne (département relativement pauvre du sud-est parisien).

23 Huit pistes sont proposées, comme le montre l'illustration suivante : 
Figure 9. Extrait du rapport Carrez synthétisant les ressources envisageables pour le financement de la SGP.

\section{Recours à l'emprunt et bilan sur la période 2010-2025}

Les nouvelles ressources d'investissement identifiées par la mission sont donc les suivantes :

\section{Bilan des ressources d'investissement}

\begin{tabular}{|l|c|}
\hline \multicolumn{1}{|c|}{ Ressource } & Recette sur la période 2010-2025 (Md€) \\
\hline Poursuite de l'effort budgétaire & 6,4 \\
Taxe sur les bureaux et autres locaux & 5,3 \\
Redevance sur la création de bureaux & 1,9 \\
Exo-Taxe Poids Lourds & 1,3 \\
Stationnement & 0,6 \\
TSE Grand Paris & 1,6 \\
Taxe de séjour additionnelle & 0,5 \\
Valorisation foncière & affectée au financement des polarités singulières \\
\hline Total & $\mathbf{1 7 , 6}$ \\
\hline
\end{tabular}

\section{Gilles Carrez, 2009}

Plusieurs propositions ne seront pas reprises, en particulier les taxes sur les usagers de la route. Pourtant, le rapport note que l'île-de-France possède un réseau autoroutier avec très peu de péages. Il envisage ainsi la création d'une éco-taxe et d'un péage urbain. La première est finalement abandonnée au niveau national suite à des manifestations en Bretagne et le second ne sera pas autorisé par la loi.

Trois propositions sont retenues : l'augmentation de la taxe sur les bureaux perçue par le conseil régional, la Taxe Spéciale d'Équipement (TSE) ajoutée à la taxe d'habitation acquittée par les ménages franciliens, et l'Imposition Forfaitaire sur les Entreprises de Réseaux (IFER). Cette dernière porte sur le matériel roulant empruntant le réseau ferré en Île-de-France et est payée par les opérateurs, notamment la RATP et la SNCF. Il est possible de voir dans cette imposition une contribution à la fois des acteurs publique participant au financement de ces deux entreprises et par les voyageurs grâce à la billetterie.

Enfin, d'autres propositions n'avaient pas été retenues dans la loi relative au Grand Paris de 2010 mais ont finalement été reprises au cours de l'année 2018 par les parlementaires. Les hausses de la taxe de séjour et de la redevance sur la création de bureaux ont été votées par les parlementaires suite aux critiques émises dans le rapport de la Cour des Comptes en décembre 2017 et sur la proposition de Gilles Carrez dans son second rapport sur le financement de la Société du Grand Paris de 2018.

Pour résumer, le mode de financement de la SGP à sa création s'appuie sur un budget composé par une contribution des entreprises via la taxe sur les bureaux, une contribution des Franciliens via la taxe d'habitation et une contribution combinée des acteurs publics et des usagers via la taxe sur les entreprises de réseau. Ce partage est assez proche de la logique régissant le fonctionnement des transports urbains en France avec d'un côté le versement transport, de l'autre la billettique et pour compléter, les collectivités territoriales.

Dans la première partie de cet article, nous avons vu que le GPE devenu NGP en 2013 était le fruit d'un ensemble de processus politiques à plusieurs échelles dont le résultat 
est un consensus autour de la construction d'une grande infrastructure de transport devant creuser une rocade de métro autour de Paris et desservir des quartiers et des clusters aux caractéristiques très différentes. Pour financer ce projet, plusieurs pistes ont été explorées et inscrites dans la loi relative au Grand Paris de 2010 avant d'être complétées petit à petit à travers les accords Etat-Région de 2011, de 2013 et des interventions ponctuelles de différents acteurs parlementaires et régionaux. La seconde partie de cet article est consacrée à l'étude du contenu de la loi relative au Grand Paris et à son application. Nous chercherons à comprendre comment le rapport Carrez de 2009 est interprété dans la loi de 2010 et comment cette loi permet aujourd'hui de dégager des ressources très conséquentes permettant d'engager simultanément les nombreux chantiers dits du Grand Paris.

\section{Le Sénat : acteur majeur de la loi relative au Grand Paris}

Afin de comprendre pourquoi et comment le rapport Carrez a influencé la loi, il faut revenir sur son élaboration depuis le dépôt du texte jusqu'à son passage au Sénat. Pour mettre en avant l'évolution du texte, nous avons regroupé dans le tableau ci-après les alinéas de l'article 9 concernant le financement du projet d'infrastructure de transport. Le tableau comporte trois colonnes : le texte initial, celui adopté en première lecture à l'Assemblée Nationale et celui adopté par le Sénat.

Les sénateurs ont modifié le texte à plusieurs égards. La première modification limite les contributions publiques à la seule contribution de l'État en écartant les collectivités territoriales. L'État devra participer au financement à hauteur de 4 mds $€$ et garantir les emprunts de la SGP. Ce montant est directement destiné au financement des investissements et non pas au fonctionnement. L'autre point important est l'ajout par l'Assemblée Nationale de la mention "sur les marchés financiers", suite à l'alinéa concernant les emprunts. Dans la pratique, la SGP s'est d'abord tournée vers la Banque Européenne d'Investissement avant d'émettre des bons sur le marché. Enfin l'ajout de deux articles par le Sénat (devenant les articles 10 et 11 dans la version finale) met en avant la nécessité pour les sénateurs de se référer au rapport Carrez (insertion de la taxe sur les bureaux) et d'y ajouter leur propre proposition (création de l'IFER).

Cette intervention du Sénat marque la volonté d'élus locaux franciliens d'éviter qu'à terme les gouvernements centraux fassent appel aux collectivités territoriales pour le financement. Cette restriction est notamment soutenue par Christian Favier, sénateur (PCF) et président du conseil général du Val-de-Marne qui avait lancé l'initiative Orbival dont le financement avait pourtant été pensé à partir d'une participation du département, du conseil régional et de l'État. Finalement, le passage au Sénat de la loi relative au Grand Paris est une occasion supplémentaire pour les élus locaux d'orienter en leur faveur le Grand Paris, en se référant à la fois au rapport de Gilles Carrez et en rappelant l'importance du soutien financier de l'État. 
Tableau 3. Evolution de la loi relative au Grand Paris (lecture accélérée), Alexandre Faure, 2019.

\begin{tabular}{|c|c|c|}
\hline Version initiale & $\begin{array}{l}\text { Adoptée par } \\
\text { l'Assemblée } \\
\text { Nationale }\end{array}$ & Adoptée par le Sénat \\
\hline $\begin{array}{l}\text { 1) Dotations publiques provenant des } \\
\text { collectivités territoriales, de l'État et de } \\
\text { l'Union Européenne ainsi que d'autres } \\
\text { organismes publiques nationaux }\end{array}$ & $\begin{array}{l}\text { 1) Sans } \\
\text { modification }\end{array}$ & $\begin{array}{l}\text { 1) Dotations en capital provenant } \\
\text { de l'Etat }\end{array}$ \\
\hline 2) Par les emprunts & 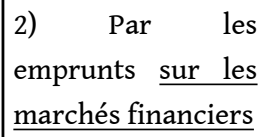 & 2) Sans modification \\
\hline $\begin{array}{l}\text { 3) Participation des aménageurs est des } \\
\text { constructeurs aux coûts des gares } \\
\text { comme le prévoit le Code de l'Urbanisme }\end{array}$ & $\begin{array}{l}3) \text { Sans } \\
\text { modification }\end{array}$ & 3) Sans modification \\
\hline $\begin{array}{l}\text { 4) Produits de la cession ou de } \\
\text { l'occupation des biens mobiliers et } \\
\text { immobiliers }\end{array}$ & $\begin{array}{l}\text { 4) Sans } \\
\text { modification }\end{array}$ & $\begin{array}{l}\text { 4) ... dont les produits des baux } \\
\text { commerciaux conclus dans les } \\
\text { gares }\end{array}$ \\
\hline 5) Redevances domaniales & $\begin{array}{ll}5) & \text { Sans } \\
\text { modification } & \end{array}$ & 5) Sans modification \\
\hline $\begin{array}{l}\text { 6) Redevances et produits des services } \\
\text { rendus }\end{array}$ & $\begin{array}{ll}6) & \text { Sans } \\
\text { modification } & \end{array}$ & 6) Sans modification \\
\hline 7) Taxes éventuellement créées & \begin{tabular}{|l}
$7)$ \\
modification
\end{tabular} & 7) Sans modification \\
\hline 8) Dons et legs & $\begin{array}{ll}\text { 8) Sans } \\
\text { modification }\end{array}$ & 8) Sans modification \\
\hline 9) Tout autres concours financiers & $\begin{array}{ll}\text { 9) Sans } \\
\text { modification }\end{array}$ & 9) Sans modification \\
\hline & & $\begin{array}{l}\underline{\text { Ajout article } 9 \text { bis : Taxe sur les }} \\
\text { bureaux, commerces et espaces de } \\
\text { stockage dans un périmètre large } \\
\underline{\text { autour des nouvelles gares }}\end{array}$ \\
\hline & & Ajout article 9 ter : IFER \\
\hline
\end{tabular}




\section{Le relèvement du versement transport entre politique de rééquilibrage et nécessité de trouver des ressources financières}

Un dernier élément d'importance concernant les ressources doit être mentionné ici. Dans le rapport Carrez, il est proposé de relevé le taux du versement transport (VT) de la zone centrale de la métropole pour dégager des ressources. En 2010, la loi ne le prévoit pas, mais très rapidement, plusieurs initiatives vont faire augmenter ce taux sur l'ensemble du territoire régional dans le but de couvrir les coûts de fonctionnement du réseau et notamment la dérive du socle.

Le VT est une singularité du modèle français de financement des politiques de transport. Il s'agit d'une recette issue d'une taxe sur les salaires des entreprises de plus de 11 salariés. Son taux d'application varie en fonction de la taille de l'agglomération et de la qualité du réseau de transport. En Ile-de-France, le taux du VT est réparti en 4 zones. La zone 1 regroupe Paris et le département des Hauts-de-Seine. La zone 2 regroupe la Seine-Saint-Denis et le Val-de-Marne. La zone 3 les secteurs urbains des départements de seconde couronne, et enfin la zone 4 les communes rurales de ces derniers. Son relèvement a été décidé pour toutes les zones de manière désordonnée depuis 2010 et le principe de ces relèvements peut apparaitre contradictoire car l'objectif initial a été abandonné en seulement quelques années.

Dans le but de financer le fonctionnement de la nouvelle infrastructure tout en finançant la dérive du socle et en poursuivant l'objectif d'un rééquilibrage entre l'est et l'ouest de la région, ce relèvement est présenté dans le rapport Carrez de 2009, comme devant être supérieur pour les secteurs les mieux dotés et les plus aisés. En 2013, les accords du NGP établissent le VT de la zone 1 à $2,7 \%$, celui de la zone 2 à $1,8 \%$ et celui de la zone 3 à 1,6 \%. En 2015, le taux est légèrement relevé (Z1 : 2,85 \%; Z2 : 1,91 \%; Z3 : $1,8 \%)$. En 2016, une nouvelle hausse concerne les zones $1(2,95 \%)$ et $3(1,91 \%)$. Dans ce cadre, les départements de Seine-Saint-Denis et du Val-de-Marne obtiennent un avantage certain en matière d'attractivité puisque le taux ne progresse pas et reste largement inférieur à celui de la zone 1. Cependant, le 14 février 2018, le STIF décide l'alignement du taux de la zone 2 sur celui de la zone 1 d'ici au $1^{\text {er }}$ juillet 2021 . Ce relèvement progressif rend caduc la volonté d'utiliser l'outil qu'est le VT pour favoriser un rééquilibrage est/ouest. Inversement, l'application d'un taux identique en SeineSaint-Denis et dans le Val-de-Marne alors que ce taux été initialement inférieur à la zone 1 va diminuer l'attractivité de ces territoires et pourrait favoriser une concentration encore plus importante des activités économiques à l'ouest de l'agglomération. Ce relèvement aura cependant comme conséquence d'apporter de plus grandes ressources au STIF pour entretenir les réseaux et investir tout en se préparant à prendre en charge le nouveau réseau dont les premiers tronçons devraient être mis en service avant les Jeux Olympiques et Paralympiques de Paris en 2024.

\section{Une augmentation constante des dépenses de la Société du Grand Paris}

Cette dernière partie est consacrée à l'application de la loi par la SGP. Pour cela, nous proposons d'étudier les rapports d'activités disponibles pour la période 2011-2017. 
Nous constatons qu'avant 2016, les ressources budgétaires de la SGP permettent de couvrir les dépenses sans recourir à l'emprunt, tandis qu'après cette date ce n'est plus le cas (figure 10). En observant les rapports d'activité, il apparaît que la SGP a souscrit un emprunt d'environ 1 mds $€$ auprès de la Banque Européenne d'Investissement et a recouru au fond de 4 mds $€$ prévu par la loi et pourvu par l'État. Enfin, comme l'avait prévu la loi, la SGP indique avoir commencé à émettre des bons de plusieurs types, que ce soit sur le marché à court terme ou des bons verts ${ }^{7}$. Les montants de ces émissions sont variables, 3 mds $€$ pour les courts termes, 5 mds $€$ pour les green bonds.

Figure 10. Résultat du cumul des recettes et des dépenses de la SGP sur la période 2011-2019, hors emprunts, en millions d'euros.

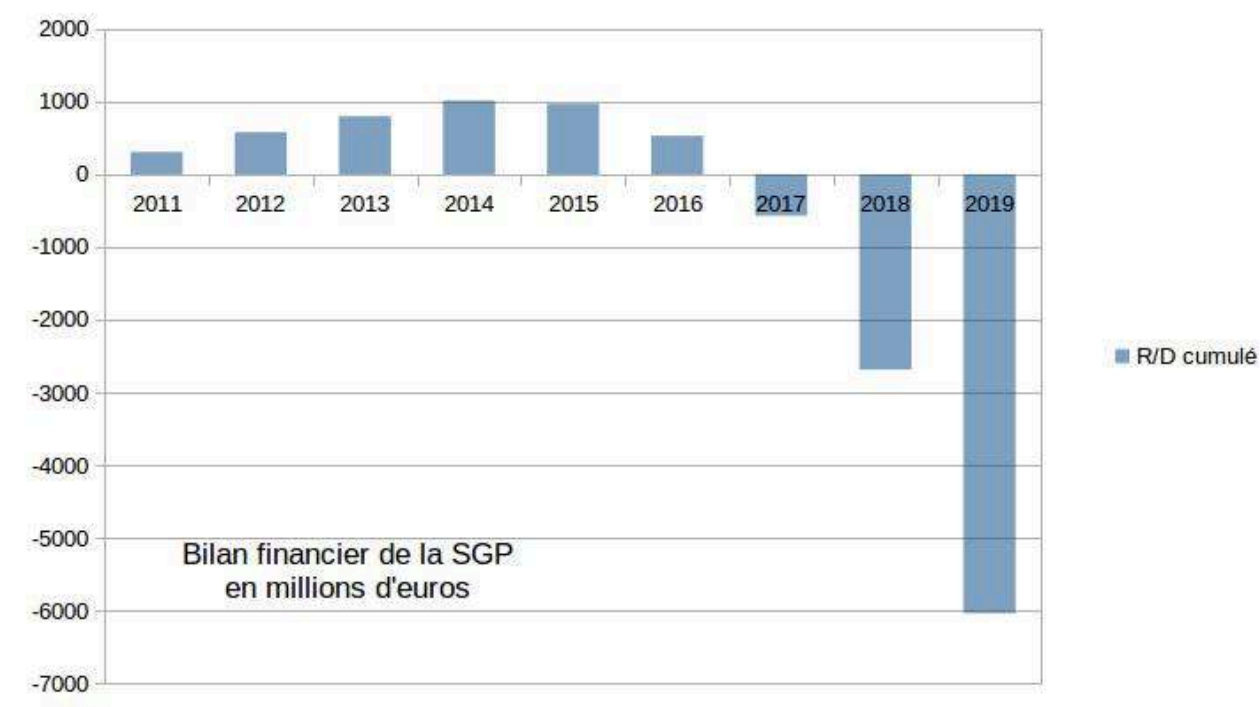

Alexandre Faure, 2019

Dans le détail, comme nous pouvons le voir sur les graphiques ci-après (figures 11, 12 et 13), les dépenses sont concentrées principalement sur l'investissement dans le GPE et dans le Plan de mobilisation (des investissements faits dans la rénovation des stations et du matériel roulant du réseau existant). La hausse des dépenses d'année en année est très importante malgré une diminution de son augmentation. L'ensemble de ces éléments montre que la SGP met en place les chantiers à un rythme élevé et avec un périmètre budgétaire conforme à l'ambition initiale. Il est difficile de savoir si ces moyens permettront de tenir les délais présentés dans la loi et annoncés par les gouvernements successifs. Le modèle de financement appliqué permet de ne pas dépendre des négociations année après année des budgets par les parlementaires et le gouvernement en reposant sur une assise fiscale suffisante pour garantir le fonctionnement et le remboursement des emprunts. Cela n'empêche pas la Société du Grand Paris d'avoir emprunté à différents acteurs publics comme la Caisse des Dépôts et des Consignations ou à la Banque Européenne d'Investissement. 
Figure 11. Dépenses cumulées inscrites dans les rapports d'activités de la SGP en prenant en compte les prévisions pour 2018 et 2019 , en millions d'euros.

Dépenses cumulées de la SGP

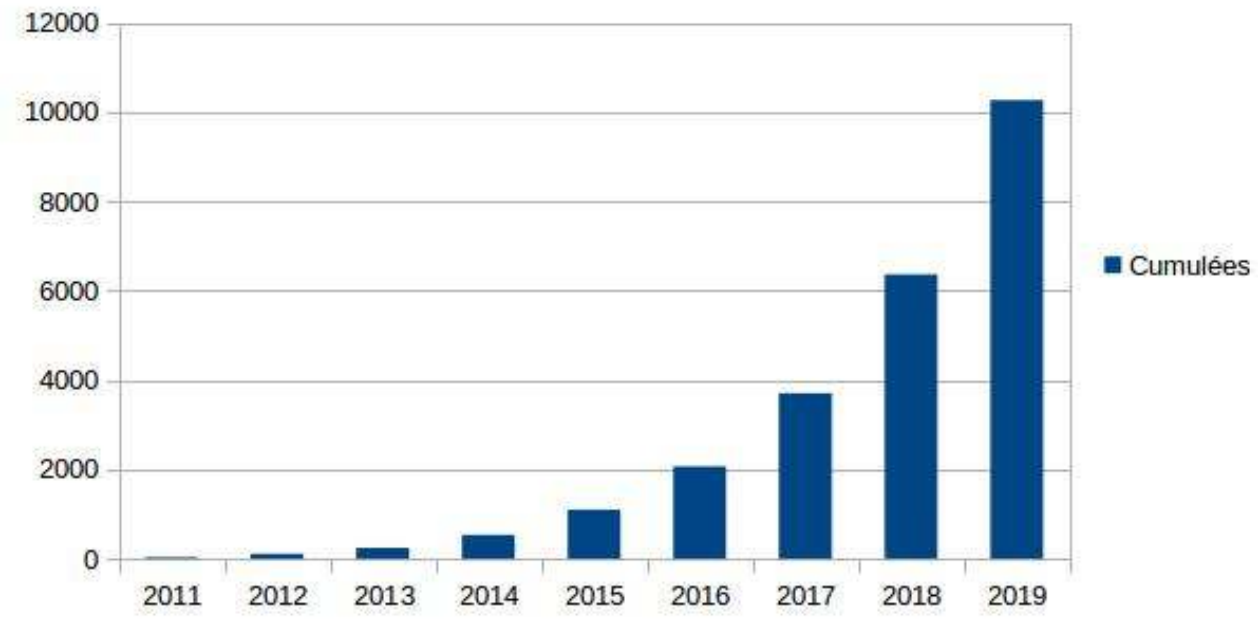

Alexandre Faure, 2019

Figure 12. Dépenses de la SGP par année, en millions d'euros.

\section{Dépenses détaillées de la SGP} en millions d'euros

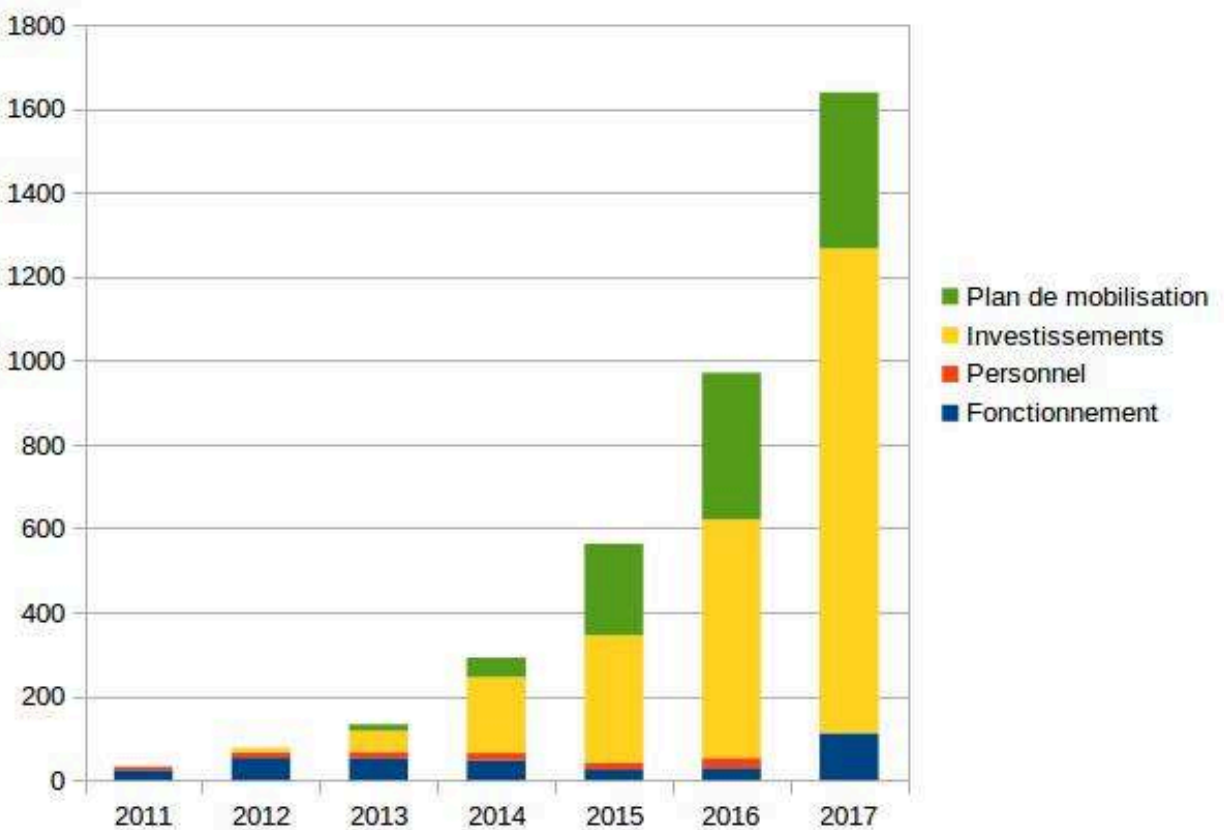

Alexandre Faure, 2019 
Figure 13. Evolution de l'augmentation des dépenses de la SGP d'une année sur l'autre.

Evolution des dépenses en pourcentage par rapport à l'année précédente

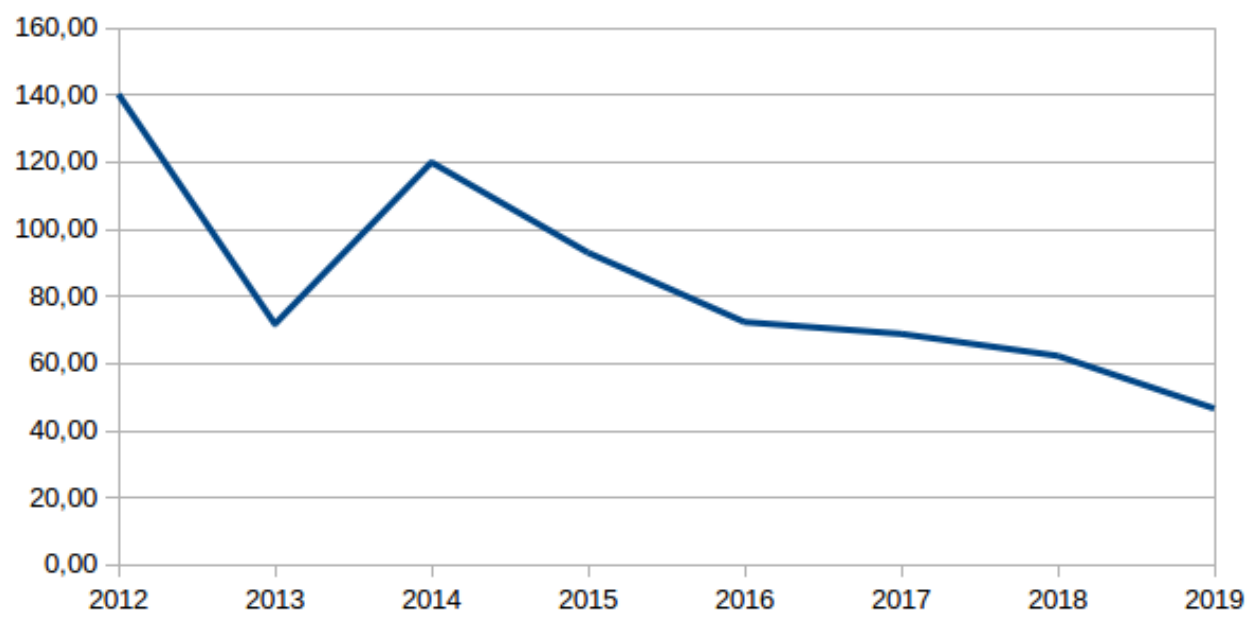

Alexandre Faure, 2019

Cette augmentation constante des dépenses et le recours aux emprunts montrent que le mode de financement proposé dans le cadre du rapport Carrez de 2009 et inscrit dans la loi relative au Grand Paris par les parlementaires et particulièrement par les sénateurs, fonctionne. Les emprunts contractés devraient être couverts par les recettes dont les niveaux ont été ajustés à la nécessité d'emprunter plus que prévu. Cette méthode conforte l'idée d'un modèle de financement régional avec une participation relativement faible de l'État (actuellement 4 mds $€$ sur les 38,5 mds $€$ inscrits dans le rapport Carrez de 2018).

Début 2016, les travaux préparatoires pour la ligne 15 sud commencent et fin 2018, toutes les stations de cette nouvelle ligne sont en constructions. Pour autant, 5 ans après l'accord du Nouveau Grand Paris, le financement du Grand Paris apparaît incertain. Dans un rapport de la Cour des Comptes rendu public fin 2017, le système d'emprunts et de réemprunts nécessaires au financement de l'infrastructure et du matériel roulant est critiqué. Le risque que les recettes fiscales de la SGP ne soient pas suffisantes pour payer les intérêts est considéré comme trop important. Gilles Carrez, auteur du premier rapport sur le financement du Grand Paris Express est alors chargé de produire un second rapport rendu en 2018. Emmanuel Macron (Président de la République depuis 2017) en visite en Seine-Saint-Denis va alors confirmer l'engagement du gouvernement pour soutenir les travaux en modifiant à la marge seulement les calendriers d'ouverture des nouvelles lignes.

\section{Conclusion : Le Grand Paris Express est l'expression de la recomposition des pouvoirs franciliens}

Le but de cet article était d'expliquer comment le financement du Grand Paris Express dont le coût excède $35 \mathrm{mds} €$ a pu être assuré au moment de la crise financière. De toute évidence, la crise a servi de tremplin pour une relance par la dépense publique et les 
infrastructures. Cette dépense a été rendue possible grâce l'impulsion du Président de la République nourrie par la volonté d'élus franciliens aux fonctions nationales.

Plusieurs éléments de ce mécanisme de financement ont été mis en lumière au cours de cet article. Tout d'abord, alors que les années 1990-2000 avaient pu donner l'impression d'un certain retrait de l'Etat et des gouvernements centraux dans l'orientation des politiques d'aménagement, l'activation de cet immense projet d'infrastructure par une initiative présidentielle vient remettre en cause ce constat. Pour autant, ce projet reflète plus un concours de circonstances particulier à l'Ile-de-France qu'une dynamique profonde de reprise en main de l'aménagement et de l'urbanisme par l'Etat. Ce projet d'infrastructure unique en France, porté initialement par le gouvernement entre 2008 et 2010, a très rapidement été pris en charge par une multitude d'élus régionaux ayant des positions nationales (parlementaires, ministres...). De fait, le Grand Paris est l'expression d'une recomposition des pouvoirs et de l'entremêlement des échelles d'action par le biais du cumul des mandats et des positions. Ensuite, nous avons montré que le GPE est le résultat d'une négociation multipartite engageant aussi bien les communes, les intercommunalités, les départements, la région, les parlementaires et le gouvernement. C'est certainement ce mélange d'institutions publiques qui caractérise ce projet d'infrastructure. Enfin, alors que le projet est la combinaison de l'action simultanée d'acteurs locaux, régionaux et nationaux, son financement, lui, est essentiellement métropolitain.

41 Ce dernier point est fondamental pour comprendre le contexte dans lequel ce projet a émergé et a été confirmé. En effet, pour que les parlementaires français acceptent un projet aussi important sur le plan budgétaire, il a fallu élaborer un mode de financement dans lequel ce n'est pas l'intégralité de la nation qui paie pour une infrastructure parisienne, mais bien les Franciliens qui paient eux-mêmes ce projet métropolitain. Ainsi, le coût de l'infrastructure est resté un débat relativement marginal contrairement à la définition de l'origine des ressources budgétaires nécessaires à sa réalisation.

Afin de mieux saisir les enjeux du financement des très grandes infrastructures de transport, une comparaison avec le projet Crossrail à Londres devrait être envisagée. Ce projet d'une grande ligne de train souterrain devant traverser Londres d'est en ouest, pour un coût estimé d'environ $15 \mathrm{mds} €$ est l'un des rares projets de la même envergure que le Grand Paris Express et dont la validation définitive remonte à 2008, année de la crise financière (un autre projet comparable serait le cas du grand métro de Moscou, mais le contexte politique et notamment l'accès aux données rendent très compliquée une comparaison avec Paris). L'infrastructure londonienne est financée par trois biais : l'entreprise de transport du Grand Londres, le gouvernement central et une taxe sur les entreprises de bureau. Deux différences avec Paris mériteraient d'être étudiées : tout d'abord la différence de statut et de pouvoir de l'entreprise de transport du Grand Londres avec le Syndicat des Transport de la région Ile-de-France dont les gouvernances et les missions sont très différentes; et ensuite la place et le poids du gouvernement central dans le financement et l'impulsion du projet. La comparaison de l'élaboration du Crossrail Act et de la loi relative au Grand Paris pourrait éclairer plus encore l'impact de la crise de 2008 sur le financement des infrastructures de transport. 


\section{BIBLIOGRAPHIE}

AALBERS M. (2012), Subprime Cities: the Political Economy of Mortgage Markets, Chichester, Malden, MA, Wiley-Blackwell.

AUZANNET P. (2018), Les secrets du Grand Paris, Paris, Hermann.

AVELINE-DUBACH N. (2008), Immobilier. L'Asie, la bulle et la mondialisation, Paris, CNRS Editions.

BAILEY S.J. (1991), « Fiscal stress: The New System of Local Government Finance in England », Sage, Urban Studies, 28, pp. 889-907.

BOUBA-OLGA O., GROSSETTI M. (2018), La mythologie CAME (Compétitivité, Attractivité, Métropolisation, Excellence) : comment s'en désintoxiquer?, HAL, hal-01724699.

BULHER T., LETHIER V. (2019), « Analysing urban policy discourses using textometry : An application to French urban transport plans (2000-2015) », Sage, Urban Studies, (en cours de publication).

CHAUVEL J. (2015), La question du Grand Paris (2001-2012), Thèse de doctorat sous la direction de Jacques Caillosse, Université Paris 2 Panthéon-Assas.

DALLIER P. (2008), Le Grand Paris : un vrai projet pour un enjeu capital, Rapport d'information $\mathrm{n}^{\circ} 262$, Sénat.

ESTÈBE P., LE GALÈS P. (2003), « La métropole parisienne : à la recherche du pilote ? », Revue française d'administration publique, ENA, 107, pp. 345-356.

FAINSTEIN S.S. (2001), The City Builders: Property Development in New York and London, 1980-2000, Lawrence, KS, University Press of Kansas.

FAURE A. (2018), Les temporalités politiques et urbanistiques du Grand Paris. Bâtir une métropole horsnorme, Thèse de doctorat sous la direction de Marie-Vic Ozouf Marignier, EHESS.

FLYVBJERG B. (2007), « Policy and Planning for Large-infrastructure Projects: Problems, Causes, Cures ", Environment and Planning B, Sage, 34, pp. 578-597.

GILLI F. (2014), Grand Paris. L'émergence d'une métropole, Paris, Les presses de SciencesPo, Paris.

GONZALEZ S., OOSTERLYNCK S. (2014), « Crisis and resilience in a financial led-city: Effects of the global crisis in Leeds ", Sage, Urban Studies, 151, pp. 3164-3179.

GOTHAM K.F. (2006), « The secondary circuit of capital reconsidered: Globalization and the US real estate sector ", The University of Chicago Press, American Journal of Sociology, 112, 1, pp. 231-275.

GUIRONNET A., HALBERT L. (2014), The financialization of Urban Development Projects: Concepts, processes, and implications, Working Paper 4, Document de travail du LATTS.

GUIRONNET A., ATTUYER K. \& HALBERT L. (2015), « Building cities on financial assets: The financialisation of property markets and its implications for city governments in the Paris cityregion ", Sage, Urban Studies, 53, 7, pp. 1442-1464.

HART T. (1993), « Transport investment and Disavantaged Regions: UK and European Policies since the 1950s », Sage, Urban Studies, 30, pp. 417-435.

HEALEY P. et al. (1992), Rebuilding the City: Property-led Urban Regeneration, London, E\&FN Spon. 
HEALEY P. (1994), « Urban policy and property development: The institutional relations of realestate development in an old industrial region ", Environment and Planning A, 26, 2, Sage, pp. 177-198.

JONAS A., GOETZ A. \& BRADY S. (2019), « The global infrastructure public-private partnership and the extra-territorial polities of collective provision: The case of regional rail transit in Denver, USA », Sage, Urban Studies, 56, pp. 1426-1447.

KRIWAN R.M. (1989), «Finance of Urban Public infrastructure », Sage, Urban Studies, 26, pp. 285-300.

LEGACY C. (2015), « Transforming transport planning in the post-political era », Sage, Urban Studies, 53, pp. 3108-3124.

LEYSHON A., THRIFT N. (2007), « The capitalization of almost everything: The future of finance and capitalism », Sage, Theory, Culture \& Society, 24, 7-8, pp. 97-115.

O'BRIEN P., PIKE A. (2018), « 'Deal or no deal?' Governing urban infrastructure funding and financing in the UK city Deals ", Sage, Urban Studies, 56, pp. 1448-1476.

O’BRIEN P., O'NEILL P. \& PIKE A. (2018), « Funding financing and governing urban infrastructures », Sage, Urban Studies, 56, pp. 1291-1303.

OREFEUIL J.P., WIEL M. (2012), Le Grand Paris. Sortir des illusions, approfondir les ambitions, Paris, Editions Scrineo.

ORUETA F.D., FAINSTEIN S.S. (2008), « The new mega-projects: Genesis and impacts », Urban Research Publications Limited, International Journal of Urban and Regional Research, 32, 4, pp. 759-767.

PANERAI P. (2008), Paris métropole, formes et échelles du Grand-Paris, Paris, Éditions de la Villette.

PRYKE M. (1994), « Looking back on the space of a boom: (Re)developping spatial matrices in the City of London », Sage, Environment and Planning A, 26, 2, pp. 235-264.

SORENSEN A., OKATA J. \& FUJII S. (2010) « Urban Renaissance as Intersification: Building Regulation and the Rescaling of Place Governance in Tokyo's High-rise Manshon Boom », Sage, Urban Studies, 47, 3, pp. 556-583.

RUTLAND T. (2010), « The financialization of urban redevelopment », Wiley, Geography Compass, 4, 8, pp. 1167-1178.

TORRANCE M.I. (2008), « Forging glocal governance ? Urban infrastructures as networked financial products », Wiley, International Journal of Urban and Regional Research, 32, 1, pp. 1-21.

VAUJOUR J. (1970), Le Plus Grand Paris, Paris, Presses Universitaires de France.

WEBER R. (2010), « Selling city futures : The financialization of urban redevelopment policy », Economic Geography, 86, 3, pp. 251-274.

WOLF-POWERS L. (2005), « Up-zoning New York Xity's mixed use neighborhoods : Property-led economic development and the anatomy of a planning dilemma ", Sage, Journal of Planning Education and Research, 24, 4, pp. 379-393.

ZENTELIN J.L. (2010), « Quel aménagement et quel réseau ferré pour les Franciliens ? Du Sdrif à la Société du Grand Paris », Les Annales de la recherche urbaine, 106, PUCA, pp. 84-99.

ZITTOUN P. (2008), « One policy for two problems : the policy-making controversies of the parisian tramway ", Taylor \& Francis, Planning theory and practice, 9, 4, pp. 459-474. 


\section{NOTES}

1. Nicolas Sarkozy, discours du 29 avril 2009 de Paris sur le projet du Grand Paris : http:// discours.vie-publique.fr/notices/097001304.html.

2. Carrez Gilles, Grand Paris. Financement du projet de transports, rapport pour le Premier ministre, 30 septembre 2009.

3. Concernant le détail des interventions pendant la discussion de la loi relative au Grand Paris, cf : https://www.senat.fr/dossier-legislatif/pjl09-123.html\#timeline-6.

4. Cour des Comptes, La Société du Grand Paris, rapport de décembre 2017.

5. Carrez Gilles, Ressources de la Société du Grand Paris, rapport pour le Premier ministre, juillet 2018.

6. Christian Blanc, discussion générale du projet de loi relatif au Grand Paris au Sénat, 6 avril 2010.

7. https://www.moodys.com/research/Moodys-attribue-une-premire-notation-dmetteurAa2Prime-1-la-Socit--PR_360653.

\section{RÉSUMÉS}

Le Grand Paris Express est un projet d'infrastructure de transport pour la région Ile-de-France voté en 2010 et dont les travaux ont débuté en 2016. Elaboré durant la crise financière, son coût élevé (environ 35 milliards d'euros) pose de nombreuses questions quant aux solutions envisagées pour dégager les ressources budgétaires nécessaires. Cet article vise à explorer le montage financier de ce projet, à en expliquer l'évolution et les origines tout en peignant un tableau du paysage politique et institutionnel ayant permis son approbation. L'enjeu est de montrer que le financement d'une telle infrastructure est un objet utile pour questionner les jeux d'acteurs, la répartition des pouvoirs et l'influence réciproque des échelles du projet. Entre initiatives gouvernementales, législation, plans stratégiques régionaux et actions départementales ou municipales, le Grand Paris s'avère être le reflet d'une dynamique de recomposition des pouvoirs publics en France et à Paris.

The Grand Paris Express is a public transport infrastructure project for the Ile-de-France region that was adopted in 2010. Elaborated during the financial crisis, lots of questions appear because of the high cost (around $€ 35$ billion) of the project about the options available to provide the budget. This article examines the financial framework of this project, explains its evolution and origins, and provides an overview of the political and institutional landscape that led to its approval. The paper will explain how the funding of this kind of infrastructure is a key element in questioning the roles of actors and the mutual influence of the project scales. Between government initiatives, legislation, regional strategic plans and departmental or municipal actions, Greater Paris is the result of a change in the structure of public authorities in France and Paris. 
INDEX

Keywords: Grand Paris, finances, public transport, Société du Grand Paris, public policies Mots-clés : Grand Paris, finances, transports publics, Société du Grand Paris, politiques publiques

\section{AUTEUR}

\section{ALEXANDRE FAURE}

Post-doctorant à la Fondation France-Japon de l'École des Hautes Études en Sciences Sociales (FFJ-EHESS), faure.alexandre@ehess.fr 